\title{
Principais marcadores moleculares
}

\author{
Principal molecular markers \\ Principales marcadores moleculares
}

\author{
Altacis Júnior de Oliveira \\ ORCID: https://orcid.org/0000-0002-6787-7160 \\ Universidade do Estado de Mato Grosso, Brasil \\ E-mail: juniormarque11@gmail.com \\ Taniele Carvalho de Oliveira \\ ORCID: https://orcid.org/0000-0002-6900-6449 \\ Universidade do Estado de Mato Grosso, Brasil \\ E-mail: taniele.carvalho@unemat.br \\ Andressa Alves Cabreira dos Santos \\ ORCID: https://orcid.org/0000-0001-6731-9575 \\ Universidade do Estado de Mato Grosso, Brasil \\ E-mail: acabreyra@gmail.com \\ Thalyson Ade Siqueira \\ ORCID: https://orcid.org/0000-0003-0155-0349 \\ Universidade do Estado de Mato Grosso, Brasil \\ E-mail: thalysonade@gmail.com \\ Willian Martins Duarte \\ ORCID: https://orcid.org/0000-0001-7180-7969 \\ Universidade do Estado de Mato Grosso, Brasil \\ E-mail: wlduarte10@gmail.com \\ Daniela Soares Alves Caldeira \\ ORCID: https://orcid.org/0000-0002-4345-3718 \\ Universidade do Estado de Mato Grosso, Brasil \\ E-mail: danielacaldeira@unemat.br \\ Marcella Karoline Cardoso Vilarinho \\ ORCID: https://orcid.org/0000-0003-1410-142X \\ Universidade do Estado de Mato Grosso, Brasil \\ E-mail: marcella.vilarinho@unemat.br \\ Mirian da Silva Almici \\ ORCID: https://orcid.org/0000-0001-9467-7060 \\ Universidade do Estado de Mato Grosso, Brasil \\ E-mail: mirianalmici@hotmail.com \\ Gustavo Ferreira da Silva \\ ORCID: https://orcid.org/0000-0002-0809-2814 \\ Universidade Estadual Paulista "Júlio de Mesquita Filho", Brasil \\ E-mail: ferreirasilvagustavo@gmail.com \\ Marco Antonio Aparecido Barelli \\ ORCID: https://orcid.org/0000-0002-6385-6733 \\ Universidade do Estado de Mato Grosso, Brasil \\ E-mail: mbarelli@unemat.br \\ Isane Vera Karsburg \\ ORCID: https://orcid.org/0000-0002-9637-0449 \\ Universidade do Estado de Mato Grosso, Brasil \\ E-mail: isane.karsburg@unemat.br
}

\section{Resumo}

Os marcadores moleculares são fenótipos moleculares provenientes de quaisquer segmentos expressos ou não de DNA que vem de herança genética e se prestam à diferenciação de um ou mais indivíduos. Pode-se classificá-lo em dois grupos: o primeiro, os marcadores são obtidos por hibridização e o segundo, os marcadores são obtidos por amplificação do DNA. Entre os identificados por hibridização estão os principais marcadores RFLP e os marcadores minissatélites, já para os marcadores por amplificação estão os mais utilizados RAPD, SCAR, STS, AFLP e SSR ou microssatélites. Diante do proposto, o presente estudo objetivou-se desenvolver uma revisão de literatura sobre os principais marcadores moleculares e suas respectivas informações. $\mathrm{O}$ trabalho busca sintetizar o conhecimento sobre os principais marcadores moleculares, pois a partir deles, é capaz de se fazer a detecção e a análise de polimorfismos genéticos que são de interesse em diversas áreas, pois podem auxiliar a compreender a base molecular de vários aspectos biológicos. Diante disso, torna-se útil o estudo por meio da revisão bibliográfica, mostrando o quão importante vem sendo os marcadores moleculares, pois podem detectar e a analisar os polimorfismos genéticos 
presentes em diversas espécies. E a partir do levantamento de periódicos dos últimos cinco anos, mostra que os marcadores RFLPs possuem o maior número de estudos feitos, seguido dos marcadores SSRs e os AFLPs. Novos estudos podem ser feitos levando em consideração mais anos para o levantamento, obtendo resultados mais concretos e detalhados.

Palavras-chave: AFLP; RFLP; SSR.

\begin{abstract}
Molecular markers are molecular phenotypes originating from any expressed or unexpressed segments of DNA that come from genetic inheritance and lend themselves to the differentiation of one or more individuals. It can be classified into two groups: the first, markers are obtained by hybridization and the second, markers are obtained by DNA amplification. Among those identified by hybridization are the main RFLP markers and minisatellite markers, while for the markers by amplification are the most used RAPD, SCAR, STS, AFLP and SSR or microsatellites. In view of this, the present study aimed to develop a literature review on the main molecular markers and their respective information. The work seeks to synthesize the knowledge about the main molecular markers, because from them, it is possible to make the detection and analysis of genetic polymorphisms that are of interest in several areas, because they can help understand the molecular basis of various biological aspects. In view of this, the study by means of literature review becomes useful, showing how important molecular markers have been, because they can detect and analyze the genetic polymorphisms present in several species. And from the survey of journals in the last five years, it shows that RFLPs markers have the largest number of studies done, followed by SSRs and AFLPs markers. New studies can be made taking into consideration more years for the survey, obtaining more concrete and detailed results.
\end{abstract}

Keywords: AFLP; RFLP; SSR.

\title{
Resumen
}

Los marcadores moleculares son fenotipos moleculares que se originan en cualquier segmento de DNA expresado o no expresado que proviene de la herencia genética y se presta a la diferenciación de uno o más individuos. Se puede clasificar en dos grupos: el primero, los marcadores se obtienen por hibridación y el segundo, los marcadores se obtienen por amplificación del ADN. Entre los identificados por hibridación destacan los marcadores RFLP y los minisatélites, mientras que para los marcadores por amplificación son los más utilizados RAPD, SCAR, STS, AFLP y SSR o microsatélites. En vista de lo propuesto, el presente estudio tuvo como objetivo desarrollar una revisión bibliográfica sobre los principales marcadores moleculares y su respectiva información. El trabajo busca sintetizar el conocimiento sobre los principales marcadores moleculares, ya que a partir de ellos es posible realizar la detección y análisis de polimorfismos genéticos que son de interés en diversas áreas, ya que pueden ayudar a comprender las bases moleculares de diversos aspectos biológicos. En vista de esto, el estudio mediante la revisión de la literatura se vuelve útil, mostrando lo importante que han sido los marcadores moleculares, ya que pueden detectar y analizar los polimorfismos genéticos presentes en varias especies. El estudio de las publicaciones periódicas de los últimos cinco años muestra que los marcadores RFLP son los que tienen el mayor número de estudios realizados, seguidos de los marcadores SSR y AFLP. Se pueden realizar nuevos estudios teniendo en cuenta más años para la encuesta, obteniendo resultados más concretos y detallados.

Palabras clave: AFLP; RFLP; SSR.

\section{Introdução}

A descoberta da técnica de PCR nos anos 1980 causou uma verdadeira revolução na pesquisa genética, possibilitando análises rápidas e práticas de um grande número de indivíduos. A técnica é baseada na síntese in vitro de milhões de cópias de um segmento específico de DNA pela ação da enzima DNA polimerase e permitindo a análise da variabilidade intra e interpopulacional com base em dados de marcadores moleculares baseados em sequências de segmentos distintas de DNA (Cavers et al., 2005; Saadaoui et al., 2017).

Os marcadores moleculares são fenótipos moleculares provenientes de quaisquer segmentos expressos ou não de DNA que vem de herança genética e se prestam à diferenciação de um ou mais indivíduos (Ferreira \& Grattapaglia, 1998). Esses marcadores podem ser originados por meio de diferentes técnicas, em que cada técnica utiliza uma estratégia particular para detectar o polimorfismo, nos quais, a diferença entre si é dada por características como a abundância no genoma, níveis de polimorfismo detectados, especificidade de locus, reprodutibilidade, requerimentos técnicos e custo (Mondini et al., 2009).

Por meio da metodologia utilizada para identificar os marcadores moleculares, pode-se classificá-lo em dois grupos: o primeiro, os marcadores são obtidos por hibridização e o segundo, os marcadores são obtidos por amplificação do DNA (Oliveira, 2021). Entre os identificados por hibridização estão os principais marcadores RFLP (Restriction Fragment Length 
Polymorphism) e os marcadores minissatélites. Já para os marcadores por amplificação estão os mais utilizados RAPD (Random Amplified Polymorphic DNA), SCAR (Sequence Characterized Amplified Regions), STS (Sequence Tagged Sites), AFLP (Amplified Fragment Length Polymorphism) e SSR ou microssatélites (Simple Sequence Repeats) (Chies et al., 2014).

O presente trabalho de revisão bibliográfica busca sintetizar o conhecimento sobre os principais marcadores moleculares, pois a partir deles, é capaz de se fazer a detecção e a análise de polimorfismos genéticos que são de interesse em diversas áreas, pois podem auxiliar a compreender a base molecular de vários aspectos biológicos. Diante do proposto, o presente estudo objetivou-se desenvolver uma revisão de literatura sobre os principais marcadores moleculares e suas respectivas informações.

\section{Metodologia}

O presente estudo é de natureza qualitativa e sendo uma revisão de literatura e com objetivo de gerar informações sobre os principais marcadores moleculares. Sendo assim, foi utilizada uma pesquisa sistemática e ordenada a partir de dados eletrônicos (periódicos científicos, documentos técnicos e livros especializados no assunto), conforme Pereira et al. (2018).

Para a análise quantitativa das publicações, foram utilizados os artigos indexados na plataforma Periódicos Capes, acessados através do site (https://www-periodicos-capes-gov-br.ezl.periodicos.capes.gov.br/index.php?) em fevereiro de 2021. A pesquisa foi realizada através da busca de artigos com as palavras RFLP, RAPD, SCAR Marcadores, STS, AFLP e SSR publicados durante o período compreendido entre 2016 e 2020.

Os dados coletados foram tabulados e analisados por meio de programas próprios para armazenamento de dados (Microsoft Office Excel 2013 e Microsoft Office Word 2013).

\section{Os Principais Marcadores Moleculares}

A criação e uso de marcadores moleculares para a detecção e exploração dos polimorfismos do DNA é um dos avanços mais significativos no campo da genética molecular, onde que esses polimorfismos de DNA se originam de um resultado de uma variação (mutação) e são geralmente referidos pelo tipo de mutação que os criou. A utilização de marcadores moleculares no DNA gera um grande número de informações distribuídos aleatoriamente ao longo do genoma. Devido a isso, são altamente valorizados em diversas áreas que envolvem genética, biologia molecular e biotecnologia.

A partir de 1980, as técnicas de biologia molecular cresceram gradativamente, tornando a manipulação do DNA uma técnica recorrente. Com isso, o uso de marcadores moleculares passou a ser usado em análises de DNA de diversas espécies, como mostra o levantamento dos últimos cinco anos mostrando a utilização de vários tipos de marcadores moleculares (Figura 1). Diante dos diversos marcadores existentes, a escolha de um ou mais deve ser conforme a metodologia e aplicação, bem como os recursos (técnico, financeiro, equipamentos) disponíveis em cada centro de pesquisa.

Os primeiros marcadores genéticos utilizados foram os marcadores morfológicos, e são utilizados até hoje, principalmente no melhoramento genético convencional, aonde são selecionadas as características desejáveis para cruzamentos de genitores promissores. Os próximos marcadores genéticos que surgiram foram os bioquímicos, tais como termenos e as isoenzimas, aonde que as primeiras moléculas utilizadas como marcadores bioquímicos foram os metabólitos secundários tais como antocianinas e compostos fenólicos, usados para distinguir entre diferentes variedades de plantas (Grover \& Sharma, 2016). E por fim, surgem os marcadores moleculares, que são muitos utilizados em estudos de biologia molecular. 
Figura 1. Estimativa aproximada do número de artigos utilizando marcadores moleculares nos últimos cinco anos.

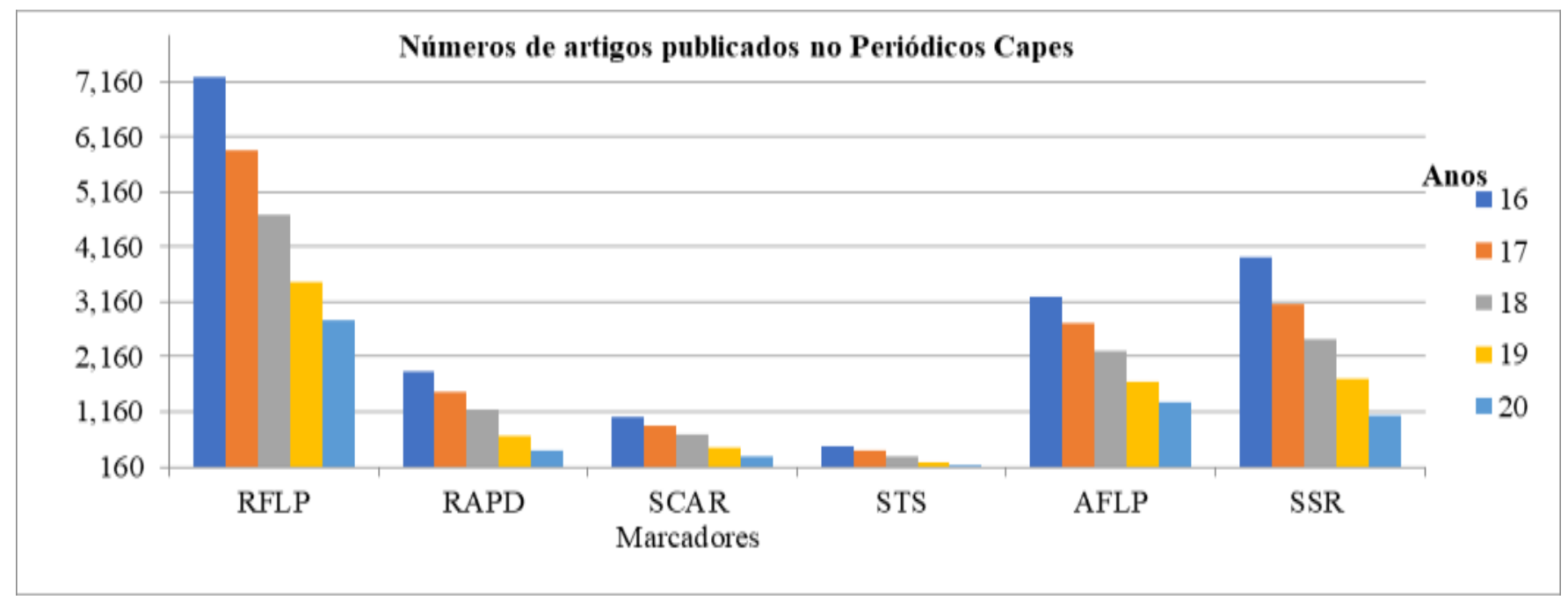

Fonte: Autores (2021).

São divididos em duas categorias principais os marcadores moleculares de DNA, sendo a primeira por hibridização (RFLP e minissatélites). E a segunda por PCR - Reação em cadeia da Polimerase (RAPD, SCAR, STS, AFLP, SSR). Também podem ser classificados de acordo com o tipo de herança alélica em dominantes e codominantes. Os marcadores codominantes propiciam distinguir indivíduos homozigotos e heterozigotos, sendo que os marcadores dominantes não são capazes, identificando apenas presença ou ausência de um determinado alelo. Diante disso, será feito um estudo mais aprofundado dos três marcadores que obtiveram maiores resultados na Figura 1, sendo eles: RFLP, SSR e AFLP.

\subsection{Marcador RFLP - Polimorfismo de Comprimento dos Fragmentos de Restrição}

Os marcadores RFLP está presente na categoria de hibridização, essa técnica é baseada em digerir todo o DNA das amostras com uma ou mais enzimas de restrição. As pequenas frações obtidas são colocadas em eletroforese de gel de agarose ou poliacrilamina, desnaturados, e transferidos para uma membrana e uma sonda de fita simples aonde é hibridizada para identificar os polimorfismos, sendo igual à técnica feita Southern Blot (Brown, 2001).

Por meio da autorradiografia, as sondas são detectadas e previamente marcadas por elementos radioativos, mas, nos dias atuais, essa sonda está sendo pouca utilizada, sendo predominantes as sondas fluorescentes ou as que participam de reações colorimétricas. Esses marcadores podem ser causados por mudanças de substituição de nucleotídeos no sítio de reconhecimento d enzima, rearranjo de DNA, inserção ou deleção.

As principais vantagens dos marcadores RFLP são a codominância e a alta reprodutibilidade. Entretanto, é uma técnica que requer um maior trabalho laboratorial, precisa de uma grande quantidade de DNA com alto grau de pureza. Além de necessitar de um conhecimento prévio da sequência para obter sondas específicas e possui ainda um custo elevado quando comparada a outros marcadores, como marcadores de sequência e microssatélites.

Konieczny e Ausubel (1993) fizeram a primeira aplicação dos marcadores RFLP para fazer mapeamento genético, mas com o passar do tempo, diversas outras aplicações foram sendo introduzidas. Esses marcadores são preferencialmente utilizados para obtenção de mapeamento genético e identificação de QTLs (Quantitative Trait Loci), em estudos com aspectos evolutivos, ou para identificar loci relacionados com características de interesse econômico.

Nos últimos anos, diversos trabalhos utilizando os marcadores moleculares RFLP vêm sendo feitos, Rohit et al. (2016) usaram o polimorfismo do comprimento do fragmento de restrição (RFLP) do gene 16S rRNA amplificado para identificar os organismos envolvidos na sepse neonatal e comparamos os achados com a hemocultura. Siqueira et al. (2017) 
usaram os marcadores RFLP para obter o perfil das comunidades microbianas, para obter a compreensão completa da diversidade microbiota. Frikha et al. (2018) usaram o RFLP para a detecção simultânea de variantes de enzimas em pacientes com leucemia aguda, na Tunísia. Nishikaku et al. (2019) aplicaram os marcadores RFLP para identificar os genótipos do vírus da leucemia bovina. Já Paulino et al. (2020) estudaram e identificaram marcadores moleculares RFLP ligados ao locus de resistência presente na cultivar Jalo Pintado 2.

\subsection{Marcador SSR - Simple Sequence Repeats ou microssatélites}

Esses marcadores possuem curtas sequências de nucleotídeos (1-6), e o seu nível de polimorfismo é dado pela quantidade distintas de repetições, provenientes de permutas desiguais que ocorrem durante a meiose ou do deslize da DNA polimerase na hora da duplicação da molécula (Morgante et al. 2002). Tais marcadores são codominantes, possuem dispersão em todo genoma e as repetições de AT são mais comuns em plantas.

Possuem alto nível de polimorfismo, potencial grande de automação, além de necessitar de baixas quantidades de DNA para iniciar a amplificação em PCR. Esses marcadores tem ampla utilização em processos de investigação genética como em estudos de diversidade genética em populações naturais e bancos de germoplasma, mapeamento e seleção assistida por marcadores e estrutura genética. A localização dos microssatélites pode estar localizada tanto em regiões que codificam para proteínas quanto em regiões que não codificam. Logo, algumas evidências mostram que em plantas, as sequências de microssatélites estão associadas, preferencialmente em regiões não codificantes do genoma (Goldstein et al. 1999).

Marcadores SSRs são obtidos com o uso de oligonucleotídeos iniciadores (primers) específicos para essas sequências adjacentes ao SSR, e por meio da PCR é possível amplificar um determinado loco e identificar os polimorfismos individuais relacionados ao número de repetições do SSR para aquele indivíduo. Logo, para cada loco de SSR há um grande conteúdo informativo, devido ser multialélico permitindo a diferenciação entre indivíduos homozigotos e heterozigotos, sendo assim, considerado um marcador codominante.

Mesmo com tantas vantagens e aplicabilidades que os SSRs possuem, existe ainda uma grande limitação no isolamento e desenvolvimento de primers específicos para cada espécie estudada. Os primeiros isolamentos e obtenção de marcadores SSRs polimórficos foram desenvolvidos por meio de técnicas laboriosas construindo bibliotecas genômicas ou bibliotecas enriquecidas para obter marcadores para a espécie que seria estudada, entretanto, nas últimas décadas tem ocorrido um progresso significativo no desenvolvimento e genotipagem de marcadores SSR, possibilitando aplicar em diversos estudos.

Uma grande quantidade de estudos mostra a utilização dos marcadores SSR. Aparecido et al. (2016) avaliaram a diversidade genética existente em uma população de eucalipto da Fibria Celulose S. A utilizando marcadores microssatélites (SSR). Guzmán et al. (2017) identificaram híbridos derivados de polinização controlada entre C. aurantifolia var. "Colimex" X C. limonvar. "Rosenberg" e seu inverso usando marcadores moleculares SSR. Hawerroth et al. (2018) utilizou o marcador SSR para caracterizar a variabilidade genética entre genótipos como copa de macieira e verificar a eficiência de discriminação dos diferentes conjuntos de caracteres. Silva et al. (2019) avaliaram a transferibilidade de 21 loci microssatélites desenvolvidos e desenhados de $P$. edulis e $P$. alata para quatro espécies silvestres de Passiflora. Já Silveira et al. (2020) estudaram a diversidade genética de populações nativas de P. cristalina por meio de marcador molecular SSR.

\subsection{Marcador AFLP - Amplified Fragment Length Polymorphism}

Os marcadores AFLP são baseados na junção dos polimorfismos gerados por enzimas de restrição e a posteriori amplificação por PCR. Essa técnica foi desenvolvida em Wageningen, Holanda e informada em uma publicação científica pela primeira vez em 1995 por Vos e colaboradores (Vos et al., 1995). O processo dessa técnica ocorre em quatro passos: Primeiro clivagem do DNA genômico, segundo - junção dos adaptadores com os fragmentos gerados na hora da clivagem do DNA, 
terceira - amplicação dos fragmentos selecionados via PCR, e quarta - divisão dos fragmentos selecionados em gel de alta resolução.

Estima-se que em cada reação de PCR há uma obtenção de 40 a 200 fragmentos, e o polimosrfismo se dá devido às mutações nos sítios reconhecidos pelas enzimas de restrição utilizadas (Grover \& Sharma, 2016). E a avaliação desses fragmentos gerados é feito por meio de presença ou ausência. Antigamente à amplificação seletiva era analisada em gel de poliacrilamida corado com nitrato de prata, hoje em dia, a amplificação é realizada via eletroforese capilar usando sequenciador, tornando a análise mais rápida e confiável.

A técnica de AFLP torna uma ferramenta útil para análise de populações segregantes, devido algumas características como: alta reprodutividade, rápida geração dos fragmentos e alta frequência de identificação de polimorfismos (Alexander et al., 2012). Ao longo do genoma os fragmentos são distribuídos e são de herança dominante, assim, o conhecimento prévio do genoma para a utilização dessa técnica é dispensável.

Diante dos marcadores moleculares existentes, os AFLPs são considerados mais reprodutíveis do que os RAPDs, sendo assim, mais confiáveis para estudos genéticos e de populações (Savelkoul et al., 1999). Entretanto, a informação de polimorfismos obtida por AFLP é menor se comparada com os resultados obtidos pelos marcadores tipo SSR (Pejic et al., 1998). Estudos utilizando a técnica de AFLP para análise do DNA pode ser feita em qualquer organismo vivo, desde microrganismos, fungos, algas, animais e plantas, como mostra os trabalhos a seguir.

Andrade et al. (2016) estimaram e caracterizaram a divergência genética entre acessos de feijão-de-vagem determinado e indeterminado do banco de germoplasma da UEL com base em marcadores AFLP. Sander et al. (2017) analisaram a diversidade e a estrutura genética de populações de Mauritia flexuosa L. f. nos biomas Cerrado (Chapada dos Guimarães - XAP) e Pantanal (Mimoso - MIM), bem como avaliaram possíveis conexões entre as populações destas áreas por meio dos marcadores AFLP. Bentes et al. (2018) estudaram a variabilidade genética de 60 isolados de Corynespora cassiicola procedentes de diferentes hospedeiras e municípios do Amazonas, usando marcadores moleculares AFLP. Wu et al. (2019) investigaram as relações genéticas de 27 acessos de E. tangutorum de diversas origens do oeste da china com o auxílio do marcador AFLP. Carvalho et al. (2020) avaliaram o grau de variabilidade intraespecífica entre as espécies de Sporothrix utilizando a técnica AFLP.

\section{Considerações Finais}

Com isso, torna útil o estudo por meio da revisão bibliográfica, mostrando o quão importante vem sendo os marcadores moleculares, pois podem detectar e a analisar os polimorfismos genéticos presentes em diversas espécies. E a partir do levantamento de periódicos dos últimos cinco anos, mostra que os marcadores RFLPs possuem o maior número de estudos feitos, seguido dos marcadores SSRs e os AFLPs. Novos estudos podem ser feitos levando em consideração mais anos para o levantamento, obtendo resultados mais concretos e detalhados.

\section{Referências}

Alexander, L. M., Kirigwi, F. M., Fritz, A. K., \& Fellers, J. P. (2012). Mapping and quantitative trait loci analysis of drought tolerance in a spring wheat population using amplified fragment length polymorphism and diversity array technology markers. Crop Science, 52(1), 253-261.

Andrade, F. A. D., Gonçalves, L. S. A., Miglioranza, É., Ruas, C. D. F., Ruas, P. M., \& Takahashi, L. S. A. (2016). Análise de AFLP na diversidade genética em acessos de feijão-de-vagem de hábito determinado e indeterminado. Acta Scientiarum. Agronomy, 38(1), 29-34.

Aparecido, M. M., Missiaggia, A. A., \& Morais, F. V. (2016). Avaliação da diversidade genética em uma população de Eucalyptus spp. Utilizando Marcador Microssatélite (SSR). Revista Univap, 22(40), 214.

Bentes, J. L. D. S., Sousa, F. M. G., Lopes, M. T. G., Valente, M. S. F., Almeida, F. V., \& Demosthenes, L. C. R. (2018). Variabilidade genética de Corynespora cassiicola isolados do Amazonas, Brasil. Arquivos do Instituto Biológico, 85. 
Brown, T. A. (2001). Southern Blotting and Related DNA Detection Techniques. Encyclopedia of Life Sceince.

Carvalho, J. A., Hagen, F., Fisher, M. C., de Camargo, Z. P., \& Rodrigues, A. M. (2020). Genome-wide mapping using new AFLP markers to explore intraspecific variation among pathogenic Sporothrix species. PLoS neglected tropical diseases, 14(7), e0008330.

Cavers, S., Degen, B., Caron, H., Lemes, M. R., Margis, R., Salgueiro, F., \& Lowe, A. J. (2005). Optimal sampling strategy for estimation of spatial genetic structure in tree populations. Heredity, 95(4), 281- 289.

Chies, S. T. T., Burchardt, P., Alves, E. M. S., Essi, L. \& Santos, E. K. (2014). O estudo da biodiversidade e evolução vegetal através de marcadores de DNA e citogenética: exemplos em Iridaceae e Poaceae. Ciência e Natura, 36, 279-293.

Ferreira, M. E., Fernández, J. N., \& Grattapaglia, D. (1998). Introducción al uso de marcadores moleculares en el análisis genético. Federal District, Brazil: Embrapa.

Frikha, R., Bouayed, N., Ben Rhouma, B., Keskes, L., \& Rebai, T. (2018). A duplex polymerase chain reaction-restriction fragment length polymorphism for rapid screening of methylenetetrahydrofolate reductase gene variants: Genotyping in acute leukemia. Journal of clinical laboratory analysis, $32(1)$, e22198.

Goldstein, D. B., \& Schlötterer, C. (1999) Microsattelites: Evolution and Applications. Oxford University Press.

Grover, A., \& Sharma, P. C. (2016). Development and use of molecular markers: past and presente. Critical Reviews in Biotechnology, (36) $290-302$.

Guzmán, M. D. J. B., Rodríguez, L. F. G., Mariscal, K. D. L. P. G., Suárez, P. A. P., \& Santos, M. O. (2017). Identificación de híbridos de Citrus aurantifolia X Citrus limon utilizando marcadores de Secuencias Simples Repetidas (SSR). Revista Mexicana de Ciencias Agrícolas, 8(6), 1397-1408.

Hawerroth, M. C., Brancher, T. L., \& Kvitschal, M. V. (2018). Dissimilaridade entre genótipos elite de macieira da Epagri com base na caracterização fenotípica e molecular. Agropecuária Catarinense, 31(3), 67-72.

Konieczny, A., \& Ausubel, F. M. (1993). A procedure for mapping Arabidopsis mutations using co-dominant ecotype-specific PCR-based markers. Plant Journal, (4) 403- 410.

Mondini, L., Noorani, A., \& Pagnotta, M. A. (2009). Assessing plantgenetic diversity by molecular tools. Diversity, 1(1), 19-35.

Morgante, M. Hanafey, M. \& Powell, W. (2002) Microsatellites are preferentialy associated with nonrepetitive DNA in plant genomes. Nature Genetics, 30, 194-200.

Nishikaku, K., Ishikura, R., Ohnuki, N., Polat, M., Aida, Y., Murakami, S., \& Kobayashi, T. (2019). Método de polimorfismo de comprimento de fragmento de restrição de PCR amplamente aplicável para genotipagem do vírus da leucemia bovina. Journal of Veterinary Medical Science, 18, 0603.

Oliveira, A. J. (2021). A cultura do Carthamus tinctorius L.: Principais usos e variabilidade genética. Research, Society and Development, 10(2), e24810212683-e24810212683.

Paulino, P. P. S., Poletine, J. P., Brondani, S. T., Barelli, M. A. A., \& da Silva, V. P. (2020). Mapeamento do gene de resistência ao Colletotrichum lindemuthianum na cultivar andina de feijão comum jalo pintado 2. Journal of Agronomic Sciences, 9, 81-107.

Pejic, I., Ajmone-Marsan, P., \& Morgante, M. (1998) Comparative analysis of genetic similarity among maize inbred lines detected by RFLPs, RAPDs, SSRs, and AFLPs. Theoretical and Applied Genetics, (97), 1248-1255.

Pereira, A. S., Shitsuka, D. M., Parreira, F. J. \& Shitsuka, R. (2018). Metodologia da Pesquisa Científica. UFSM NTE.

Rohit, A., Maiti, B., Shenoy, S., \& Karunasagar, I. (2016). Polimorfismo de comprimento de fragmento de restrição de reação em cadeia da polimerase (PCRRFLP) para diagnóstico rápido de sepse neonatal. The Indian Journal of Medical Research, 143(1), 72.

Saadaoui, E., Martín, J. J., Tlili, N., \& Cervantes, E. (2017). Castor bean (Ricinus communis L.): Diversity, seed oil and uses. Oil Seed Crops: Yield and Adaptations under Environmental Stress, 19 - 33.

Sander, N. L., Tiago, A. V., de Arruda, J. C., Carniello, M. A., Silva, M. T. P., Rossi, A. A. B., \& da Silva, C. J. (2017). Diversidade genética e estrutura populacional de Mauritia flexuosa L. f. no Cerrado e no Pantanal. Boletim do Museu Paraense Emílio Goeldi-Ciências Naturais, 12(2), $209-219$.

Savelkoul, P. H. M., Aarts, H. J. M., \& Haas, J. (1999) Amplified-Fragment Length Polymorphism Analysis: the State of an Art. Journal of Clinical Microbioloy, 37, 3083-3091.

Silva, L. P., Marostega, T. N., Gilio, T. A. S., Serafim, M. E., Araújo, K. L., \& Neves, L. G. (2019). Transferibilidade de marcadores microssatélites para espécies silvestres de maracujazeiro. Revista de Ciências Agrárias Amazonian Journal of Agricultural and Environmental Sciences, 62.

Silveira, G. F., Souza Arenas, M. D., Varella, T. L., Schmitt, K. F. M., Lima, J. A., da Silva, B. M., \& Rossi, A. A. B. (2020). Estrutura Populacional e Variabilidade Genética em Populações Nativas de Passiflora cristalina Vanderpl. \& Zappi, em Fragmentos Florestais no estado de Mato Grosso. Brazilian Journal of Development, 6(11), 87328-87341.

Siqueira, J. F, Sakamoto, M., \& Rosado, A. S. (2017). Perfil da comunidade microbiana usando polimorfismo de comprimento de fragmento de restrição terminal (T-RFLP) e eletroforese em gel de gradiente desnaturante (DGGE). Oral Biology, 139-152.

Vos, P., Hogers, R., \& Bleeker, M. (1995) AFLP: a new technique for DNA fingerprinting. Nucleic Acids Research, 21, 4407-4414.

Wu, W. D., Liu, W. H., Sun, M., Zhou, J. Q., Liu, W., Zhang, C. L., \& Ma, X. (2019). Genetic diversity and structure of Elymus tangutorum accessions from western China as unraveled by AFLP markers. Hereditas, 156(1), 1-13. 